\title{
Behavioral effects of isolation in the rat
}

\author{
K. E. MOYER AND JAMES H. KORN \\ CARNEGIE INSTITUTE OF TECHNOLOGY
}

\begin{abstract}
Abstraet
Male albino rats were raised either invidually $(\mathrm{N}=13)$ or in groups $(\mathrm{N}=12)$ from weaning at 21 days of age until they were tested at 111 days of age. Experimenter ratings and a "timidity" test showed the isolated Ss to be more emotional than group-raised Ss. No significant differences were found on startle or activity tests.

\section{Problem}

According to Hatch et al (1963), after being housed for three months in an individual cage, "the isolated rat is a nervous, aggressive intractable animal." Objective tests, such as the open field and the emergence or "timidity" test (Ader \& Friedman, 1964; Hahn, 1965; Stern, et al, 1960) also support the conclusion that isolated rats are more emotional than group-housed rats. The purpose of the present study was to replicate some of the findings with respect to emotionality and to determine, by means of a startle test, whether the general level of arousal is higher in animals which have been isolated.
\end{abstract}

\section{Method}

At weaning (21 days of age), 30 male albino rats were assigned to either a group ( 8 or $7 \mathrm{Ss} /$ cage) or an individual cage using a split litter control. The group cages measured $9-1 / 2$ by 7 by 24 in and the single cages were 7 by 7 by $9-1 / 2$ in. As a result of disease or experimenter error two isolated and three group-housed Ss were lost before testing. Animals were housed under standard laboratory conditions with food and water ad lib in external hoppers and bottles.

At 111 days of age, Ss in group cages were put into single cages which were randomly distributed among the cages of Ss which had been raised in isolation. Each cage was individually numbered, but in no way could the cages be differentiated by group. About $1 \mathrm{hr}$. later, an $\mathrm{E}$ who had not taken part in assigning grouped Ss to single cages rated the Ss on a 6-point scale for emotionality. The test involved picking up the $\mathrm{S}$ (gloved hand over the S's back), placing it on an adjacent table and then replacing it in its cage. The following ratings were applied: $0-\mathrm{S}$ made no attempt to escape from $\mathrm{E}$ and did not vocalize or jump; 1 -startle response on first attempt to handle $\mathrm{S}$, but no vocalization or vigorous escape attempts; 2 - startle and vocalization; 3 - startle, greater vocalization and vigorous escape attempts; 4 -extreme vocalization and struggling, defecation; 5-same as 4 with biting of E's glove.

About $2 \mathrm{hr}$. later, the first of three dependent variables was administered, the other two following at 24 $\mathrm{hr}$. intervals. Each $\mathrm{S}$ was given the following tests in the order listed:
Startle: S's responses to five gun-shot stimuli (for description of apparatus and procedure see Moyer, 1963). At this time an $\mathrm{E}$ who did not know to which groups the Ss had been assigned rated them on a dichotomous scale of "emotional-nonemotional." Runway Activity: This was a modified open-field test in which ambulation and defecation were measured during a $4 \mathrm{~min}$. period in a runway 6 by 8 by 42 in, painted gray with a white Formica floor. It was divided into 10 sections by lines painted on one wall. Timidity: Ss were placed in a small start box 5 by $6-1 / 2$ by 8 in separated by a guillotine door from a larger (34 by 11 by 16-1/2) compartment. The apparatus was made of wood painted light gray inside and had a floor of $1 / 8$ in brass rods spaced $1 / 2$ in apart. The larger compartment was illuminated by two 25-w light bulbs. After the guillotine door was opened the latency of emergence of S's body (excluding the tail) was timed by a stop watch. If $S$ did not leave the start box within 300 sec., it was returned to its home cage.

\section{Results and Diseussion}

Emotionality rating: Of the isolated Ss, 10 out of 13 had ratings of 2 or better, while only two of 12 groupraised Ss rated that high. The difference was significant (Mann-Whitney $U=27, p<.02,2$-tail) and supports the observation of Hatch et al (1963) that isolated animals are more emotional.

Startle. The mean startle response for the isolated and grouped Ss was 6.7 and $7.7 \mathrm{~mm}$ respectively (nonsignificant difference). In the dichotomous rating of emotionality, no grouped Ss and eight isolated Ss were rated as emotional ( $p<.01$, Fisher test). Although isolated Ss have been found to exhibit a larger startle response to handling (Ader \& Friedman, 1964), they did notdo so in this situation even though they were still rated as being more emotional.

Runway Test. The mean activity (number of lines crossed) during $4 \mathrm{~min}$. was 85 for grouped Ss and 72 for isolates. Although this difference was not significant, it was in the same direction as found in other studies (Ader \& Friedman, 1964; Stern et al, 1960). Mean defecation scores during the runway test were: isolated Ss - 2.4; grouped Ss - $4.0(\mathrm{U}=45, \mathrm{p}<.10)$. The investigations of Ader and Stern also produced this paradoxical result: isolated rats, which appear to be more emotional on other measures, are less emotional than grouped Ss when this behavior is defined in terms of defecation in a novel situation. However, none of these studies, including the present one, measured home cage defecation or defecation in transit from home cage to test situation. 
It is possible that isolated Ss defecate less in general than do grouped Ss. It also could be that isolates are really more emotional and the stimulus of being picked up and transported by $\mathrm{E}$ produces a large emotional response (defecation) which leaves less waste material to be deposited in the test situation.

Timidity. Mean emergence (whole body) latencies for the isolated and grouped Ss were 62 and $10 \mathrm{sec}$. respectively $(\mathrm{U}=38, \mathrm{p}<.05)$, again indicating greater emotionality for the isolated Ss and supporting the results of other studies (Ader \& Friedman, 1964; Hahn, 1965).

In general, these results confirm the hypothesis of greater emotionality in rats which have been housed individually for a relatively long period of time. However, as Hahn (1965) has pointed out, the term "emotionality" must be used with caution. Stern et al (1960) suggested that the exploratory behavior of isolated Ss (as in the emergence test) was restricted because of prior restrictions on such behavior in their home cages and that group housing has a "gentling" effect which is probably due to the greater amount and variety of sensory experiences to which these Ss had been exposed. In contrast to exploratory behavior, it has been found (Hahn, 1965; Stern et al, 1960) that the response to stress is greater (more emotional) in group-housed Ss than in isolates following prolonged restraint, a situation which has greater similarity to the previous history of the isolated Ss. Thus, it appears that similarity of the housing situation to the test situation may be a critical factor in determining which animals will be more "emotional."

\section{References}

Ader, R., \& Friedman, S. B. Social factors affecting emotionality and resistance to disease in animals: IV. Differential housing, emotionality and Walker 256 carcinosarcoma in the rat. Psychol. Rep., $1964,15,535-541$.

Hahn, W. W. Some effects of group size on behavior and physiology of the rat. J. psychosom. Res., 1965, 8, 455-465.

Hatch, A., Wiberg, G. S., Balzas, T., \& Grice, H. C. Long-term isolation stress in rats. Science, 1963, 142, 507.

Moyer, K. E. Startle response: Habituation over trials and days, and sex and strain differences. J. comp. physiol. Psychol., $1963,56,863-865$.

Stern, J. A., Winokur, G., Eisenstein, A., Taylor, R., \& Sly, M. The effect of group vs. individual housing on behavior and physiological responses to stress in the albino rat. J. psychosom. Res., 1960, 4, 185-190.

\section{Note}

1. This investigation was supported in part by a research grant M-1298 from the National Institutes of Health, United States Public Health Service to K. E. Moyer. 